\title{
Timing mirror structures observed by Cluster with a magnetosheath flow model
}

\author{
V. Génot ${ }^{1}$, L. Broussillou ${ }^{1}$, E. Budnik ${ }^{2}$, P. Hellinger ${ }^{3,4}$, P. M. Trávníček ${ }^{3,4,5}$, E. Lucek $^{6}$, and I. Dandouras ${ }^{1}$ \\ ${ }^{1}$ IRAP, UMR5277, CNRS - Université de Toulouse (UPS), Toulouse, France \\ ${ }^{2}$ Noveltis, 2 Avenue de 1'Europe, 31520 Ramonville Saint-Agne, France \\ ${ }^{3}$ Astronomical Institute, ASCR, Prague, Czech Republic \\ ${ }^{4}$ Institute of Atmospheric Physics, Prague, Czech Republic \\ ${ }^{5}$ Space Sciences Laboratory, University of California Berkeley, CA, USA \\ ${ }^{6}$ Space and Atmospheric Physics Group, Blackett Laboratory, Imperial College, London, UK
}

Received: 24 February 2011 - Revised: 3 August 2011 - Accepted: 25 September 2011 - Published: 24 October 2011

\begin{abstract}
The evolution of structures associated with mirror modes during their flow in the Earth's magnetosheath is studied. The fact that the related magnetic fluctuations can take distinct shapes, from deep holes to high peaks, has been assessed in previous works on the observational, modeling and numerical points of view. In this paper we present an analytical model for the flow lines and velocity magnitude inside the magnetosheath. This model is used to interpret almost 10 years of Cluster observations of mirror structures: by back tracking each isolated observation to the shock, the "age", or flow time, of these structures is determined together with the geometry of the shock. Using this flow time the evolutionary path of the structures may be studied with respect to different quantities: the distance to mirror threshold, the amplitude of mirror fluctuations and the skewness of the magnetic amplitude distribution as a marker of the shape of the structures. These behaviours are confronted to numerical simulations which confirm the dynamical perspective gained from the association of the statistical analysis and the analytical model: magnetic peaks are mostly formed just behind the shock and are quickly overwhelmed by magnetic holes as the plasma conditions get more mirror stable. The amplitude of the fluctuations are found to saturate before the skewness vanishes, i.e. when both structures quantitatively balance each other, which typically occurs after a flow time of 100-200 s in the Earth's magnetosheath. Comparison with other astrophysical contexts is discussed.
\end{abstract}

Keywords. Magnetospheric physics (Magnetosheath) Space plasma physics (Kinetic and MHD theory; Waves and instabilities)

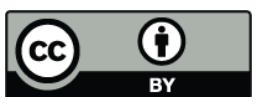

Correspondence to: V. Génot (vincent.genot@irap.omp.eu)

\section{Introduction}

Mirror modes have been studied extensively in recent years both by theoretical and observational means. Despite this activity a global physical description is still lacking. Mirror modes are mostly studied in the framework of the mirror instability which develops in anisotropic plasma $\left(T_{\perp} / T_{\|}>1\right)$ with large $\beta$ although alternative views exist (Stasiewicz, 2004). Theoretical studies are generally confined to a certain regime of parameters: close/far from instability threshold (Hellinger, 2007; Califano et al., 2008) or type of approach (linear Hall MHD (Hau et al., 2005), Landau fluid model retaining finite Larmor radius corrections (Passot et al., 2006), kinetic theory (Hasegawa, 1969; Hall, 1979). Observational works (Kaufmann et al., 1970; Gary et al., 1993; Fazakerley and Southwood, 1994; Schwartz et al., 1996; Bavassano Cattaneo et al., 1998; Horbury et al., 2004; Lucek et al., 2001), on the other hand, inherently lack the dynamical view necessary for a full understanding of the processes at work. Indeed mirror structures evolve significantly during their travel from the bow shock, where the instability is supposedly triggered, to the depletion layer close to the magnetopause or flank magnetosheath. In the following we propose to infer the history of observed plasma structures from an analytical magnetosheath model. By assuming that mirror structures are created at, or close to the shock, we use this model to gain an understanding of the structure evolution.

The rationale in the development of a "light" model is its ability to be used in the context of large scale statistical analysis and in the perspective of extending the result obtained in a previous paper (Génot et al., 2009a). We base our approach on the one developed in Tátrallyay and Erdös (2002) and Tátrallyay et al. (2008), two studies which make use of a magnetic field model initially exposed in Kobel and Flückiger (1994) (hereafter KF model). In the following

Published by Copernicus Publications on behalf of the European Geosciences Union. 


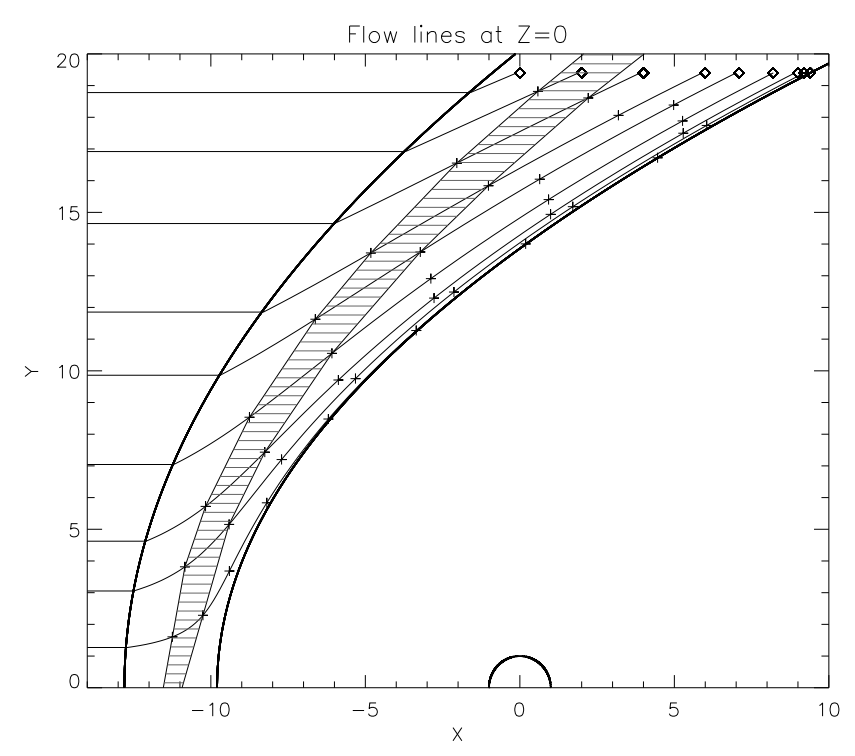

Fig. 1. Flow lines in the $\mathrm{XY}$ plane with parabolic models for the shock $\left(R_{\mathrm{bs}}=12.8 R_{\mathrm{E}}\right)$ and the magnetopause $\left(R_{\mathrm{mp}}=9.8 R_{\mathrm{E}}\right)$. These lines corresponds to the magnetic field lines of the KF model for an IMF aligned with the Sun-Earth line. Crosses are superposed on each line and correspond to elapsed times (flow times) of $100 \mathrm{~s}$, $200 \mathrm{~s}, 300 \mathrm{~s}, \ldots$ from the shock crossing. The striped region corresponds to flow times in the $100 \mathrm{~s}-200 \mathrm{~s}$ range $\left(v_{\mathrm{SW}}=400 \mathrm{~km} \mathrm{~s}^{-1}\right)$.

we shall use this model to obtain the flow lines pattern in the magnetosheath. However we shall refine our approach concerning the velocity distribution to include 3-D aspects to be used in the observational context. More refined analytic models of magnetosheath magnetic field than the KF model have been proposed. Kallio and Koskinen (2000) developed a semi-empirical 3-D magnetosheath model. They use parabolic coordinates and an analytic expression for the 3-D velocity field from which the magnetic field is derived assuming the frozen-in-the-flow condition. An analytic magnetospheric model must also be supplied. Romashets et al. (2008) developed a model also considering parabolic bow shock and magnetopause. The magnetic field is determined from a vector potential after specifying RankineHugoniot relations at the shock and a dipolar magnetospheric field. Analytical expressions obtained in these studies are far less tractable than those from Kobel and Flückiger (1994)'s model which will be preferred in the present paper as it fits our need of computational simplicity. The aim of the paper is indeed not to accurately model the magnetosheath magnetic and velocity fields but to make use of them in a systematic procedure.

To locate mirror structures in the magnetosheath, previous works (including Génot et al., 2009a) made use of a fractional distance $F$ in the magnetosheath between the magnetopause $(F=0)$ and the shock $(F=1)$ which does not take into account the history of the plasma parcel journey in the magnetosheath. Indeed this parameter $F$ was calculated by tracing a ray from the Earth centre and, successively, trough a modeled magnetopause, the observed point and a modeled shock (see for instance Verigin et al., 2006).

The aim in developing an analytic magnetosheath model is two-fold:

1. the flow lines allows a correct back tracking from an observation to the originating shock crossing, enabling to determine the shock geometry by calculating the shock angle (w.r.t. the IMF orientation),

2. the velocity magnitude distribution inside the magnetosheath, together with the tracking to the shock, allows to compute the flow time between the shock crossing and a given observation in the magnetosheath.

This applies to a plasma parcel or blob which propagates with a velocity negligible with respect to the flow velocity. Non propagating mirror modes are very well suited in that respect.

In the following section of the paper we expose our magnetosheath model which is used to analyse observations. Analytic developments are deferred to the Appendix. In the third section we present the magnetosheath observations and the way mirror structures are selected. The fourth section before the conclusion is devoted to the discussion of the results of the statistical analysis taking into account the novel information provided by the model.

\section{Magnetosheath model}

The velocity is the crucial quantity to model: the vector enables to computes the flow lines in the magnetosheath, whereas the magnitude is necessary to compute the flow time between the shock and a given position or observation. Both aspects are now addressed.

\subsection{Flowlines}

Similarly to the work of Tátrallyay and Erdös (2002) and Tátrallyay et al. (2008) the flow lines are calculated from the KF model. The KF model is an analytic model originally developed for the magnetosheath magnetic field. It is based on parabolic shapes for the shock and magnetopause and takes the IMF as input. Specifically the magnetic field lines of the KF model for the case $\boldsymbol{B}_{\mathrm{IMF}}=\left(B_{\mathrm{x}}, 0,0\right)_{\mathrm{GSE}}$ are identical to the plasma flow lines (i.e. when $\boldsymbol{B}_{\mathrm{IMF}}$ is normal to the shock at the subsolar point). Assuming that the flow properties in the magnetosheath do not depend on the IMF orientation, the flow lines for all IMF orientation are exactly given by the magnetic field lines for this particular case $\boldsymbol{B}_{\mathrm{IMF}}=\left(B_{\mathrm{X}}, 0,0\right)_{\mathrm{GSE}}$. This is illustrated on Fig. 1 . The flow lines satisfy the relation $v_{\mathrm{x}, \mathrm{y}, \mathrm{z}} / v_{\mathrm{z}, \mathrm{x}, \mathrm{y}}=B_{\mathrm{x}, \mathrm{y}, \mathrm{z}} / B_{\mathrm{z}, \mathrm{x}, \mathrm{y}}$. 


\subsection{Flowtime}

To compute the velocity magnitude requires more caution. We start from the stationary situation for which

$\operatorname{div}(\rho \boldsymbol{v})=\operatorname{div} \boldsymbol{B}=0$,

and we look for solution of the form

$\rho \boldsymbol{v}=C \boldsymbol{B}+\boldsymbol{r o t} \boldsymbol{\Phi}$

where $C$ is a function of the position and $\boldsymbol{\Phi}$ a vector. Following the above hypothesis (the flow lines are identical to the magnetic field lines of the KF model for $\boldsymbol{B}_{\mathrm{IMF}}=$ $\left.\left(B_{\mathrm{X}}, 0,0\right)_{\mathrm{GSE}}\right)$ we shall assume that $\boldsymbol{r o t} \boldsymbol{\Phi}=\mathbf{0}$. To satisfy Eq. (1) $C$ must also be constant along a flow line, i.e. $\nabla C \cdot B=0$. Therefore the equation

$\rho \boldsymbol{v}=C \boldsymbol{B}$

must be satisfied along a given flow line and in particular just behind the shock on the magnetosheath side. $C$ may therefore be written

$C=\rho_{\mathrm{bs}} \frac{v_{\mathrm{x}}}{B_{\mathrm{x}}}$

where $\rho_{\mathrm{bs}}$ is the magnetosheath density just behind the shock and $v_{\mathrm{x}}$ and $B_{\mathrm{x}}$ are the components of the velocity and magnetic field vectors downstream of the shock which can be determined by the Rankine-Hugoniot relations (see Appendix A for details). Finally our modeled velocity is given by

$\boldsymbol{v}=\frac{\rho_{\mathrm{bs}}}{\rho} \frac{v_{\mathrm{x}}}{B_{\mathrm{X}}} \boldsymbol{B}$

At this stage the density profile in our modeled magnetosheath is unknown. We envisaged three approaches: (1) a constant density, (2) a density satisfying the condition $\nabla \rho$. $\boldsymbol{v}=0$, i.e. the density is constant along a flow line, and (3) a specific density profile. The two first approaches are equivalent to considering the simpler expression $\boldsymbol{v}=\boldsymbol{C} \boldsymbol{B}$ in lieu of Eq. (3) and therefore $\boldsymbol{v}$ is given by

$\boldsymbol{v}=\frac{v_{\mathrm{x}}}{B_{\mathrm{X}}} \boldsymbol{B}$

Although this gives satisfactory results for the general velocity magnitude, it has the tendency to underestimate the velocity close to the magnetopause. To circumvent this issue we shall use the third approach by considering a density profile with a constant value from the shock to a distance close to the magnetopause and a sharp decrease (of a factor 1.25) thereafter. Such a configuration models the depletion layer close the magnetopause. Our reduction factor is conservative compared to the values between 1.5 and 2 which are indeed observed in the subsolar region (Paschmann et al., 1978). However it should be noted that, in our crude model, this value is constant along the entire magnetopause whereas the 1.5-2 factors are observed at the subsolar point where the

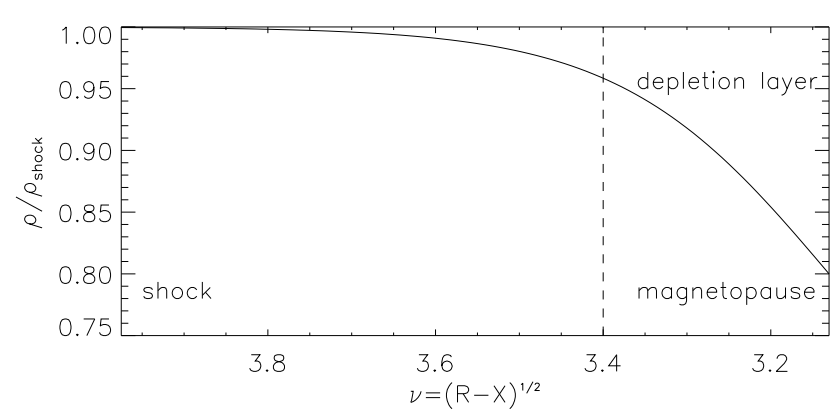

Fig. 2. Density profile in the magnetosheath exhibiting a depletion layer close to the magnetopause (with a $20 \%$ decrease). The coordinate system is the one used in the KF magnetic field model ( $R_{\mathrm{bs}}=12.8 R_{\mathrm{E}}$ and $R_{\mathrm{mp}}=9.8 R_{\mathrm{E}}$ are used).

depletion process is enhanced (by increased magnetic pileup). Smaller values are expected on the flanks. We chose the following expression where $\rho_{\mathrm{mp}}$ is the magnetosheath density close to the magnetopause:

$\frac{\rho}{\rho_{\mathrm{bs}}}=\frac{\rho_{\mathrm{mp}}}{\rho_{\mathrm{bs}}}+\left(1-\frac{\rho_{\mathrm{mp}}}{\rho_{\mathrm{bs}}}\right) \times \tanh \left(\alpha \frac{v-v_{\mathrm{mp}}}{v_{\mathrm{bs}}-v_{\mathrm{mp}}}\right)$

with $\rho_{\mathrm{mp}} / \rho_{\mathrm{bs}}=0.8, \alpha=4$ and where $\nu_{\mathrm{bs}}$ and $\nu_{\mathrm{mp}}$ are the parabolic parameters associated to the shock and the magnetopause locations respectively. They are defined in the $\mathrm{KF}$ model as $\nu_{\mathrm{bs}}=\sqrt{2 R_{\mathrm{bs}}-R_{\mathrm{mp}}}$ and $\nu_{\mathrm{mp}}=\sqrt{R_{\mathrm{mp}}} . v$ is the parabolic coordinate which varies between $\nu_{\mathrm{bs}}$ and $\nu_{\mathrm{mp}}$ and $R_{\mathrm{bs}}$ and $R_{\mathrm{mp}}$ are the stand-off positions of the shock and the magnetopause respectively. The density profile is shown on Fig. 2.

With the above assumptions it is possible to compute the velocity magnitude in the 3-D magnetosheath. The velocity contours in the XY plane are shown on Fig. 3. Consistently the velocity strongly decreases close to the nose of the magnetopause and increases in the flank of the magnetosheath. These contours compare very well with those obtained from gas dynamics models, for instance the one by Spreiter et al. (1966) (for which the shock and magnetopause surfaces are not parabolic). The comparison with their Fig. 7 (computed for $M_{\mathrm{A}}=8$ and $\gamma=2$ ) shows that in our model contours are more curved in the middle magnetosheath which implies slightly smaller velocities compared to gas dynamics values. In both models the Y-axis intersects the $v / v_{\mathrm{SW}}=0.75$ contour in a similar region ( $v_{\mathrm{SW}}$ is the solar wind velocity).

To follow the evolution of convected mirror structures, which are inherently linked to the growth of the mirror instability, the cyclotron gyroperiod is the relevant characteristic time. Similarly to what is done in hybrid simulation modeling (see the discussion in Sect. 4) this characteristic time is used for normalization, by evaluating it in the magnetosheath side just behind the shock (using our flowline model, the IMF magnitude and the shock parameter $R$ described in Appendix A). 


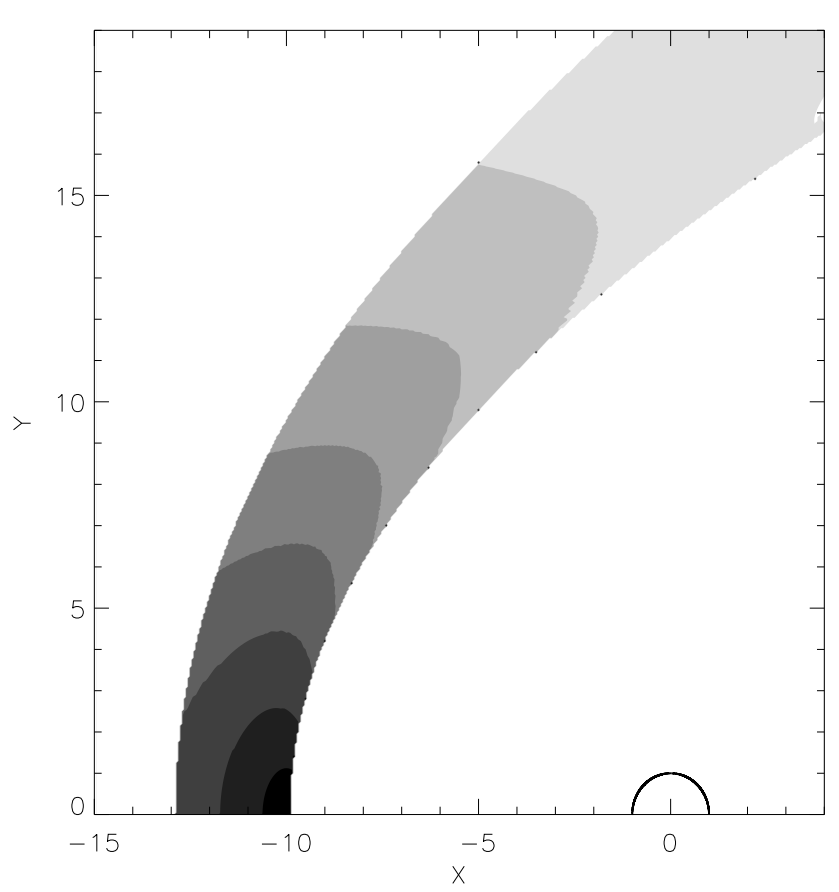

Fig. 3. Velocity contours in the $X Y$ plane with parabolic models for the shock $\left(R_{\mathrm{bs}}=12.8 R_{\mathrm{E}}\right)$ and the magnetopause $\left(R_{\mathrm{mp}}=9.8 R_{\mathrm{E}}\right)$. Each contour value (from 0.1 to 0.7 ) represents the ratio of the magnetosheath velocity $v$ to the solar wind velocity $v_{\mathrm{SW}}$ (the upper one is 0.7 ).

Finally the flowtime $T$ along a given magnetosheath flowline and normalized to the "magnetosheath side" shock gyroperiod is given by the integral

$T=\frac{q}{m} B_{\mathrm{MS}}\left(\right.$ shock) $\int_{\text {shock }}^{\text {observation }} \frac{d s}{V(s)}$

where $B_{\mathrm{MS}}$ (shock) is the magnetosheath magnetic field just behind the shock.

\subsection{Shock angle}

Once the trajectory of a plasma blob is tracked back to the shock along its associated flowline, it is possible to compute the shock normal and therefore the angle between this normal and the IMF ( $\theta_{\mathrm{Bn}}$ in the following).

\section{Observations}

Data are selected is a similar way to the one described in Génot et al. (2009a). We summarize the process below. The novelty concerns the determination of (1) the flowtime since the shock crossing and (2) the shock angle for each event using the model described above.

\subsection{Data}

Almost 10 years (from 1 February 2001 to 31 December 2009) of the Cluster mission are considered. Cluster 1 and Cluster 3 magnetic fields (FGM, Balogh et al., 2001) and on-board calculated ion moments (from the HIA experiment on the CIS instrument, Rème et al., 2001) data are used at $4 \mathrm{~s}$ resolution. ACE plasma and IMF data are also used to determine the magnetopause and bow shock positions, using Shue et al. (1997) and Verigin et al. (2006) models respectively. From these data, we construct a "magnetosheath" dataset which is further reduced to a "magnetosheath - mirror" dataset as it is explained below. Based on the same dataset a statistical analysis of mirror mode occurrence (localisation, fluctuation amplitude) has recently been published by Génot et al. (2009b). The results compare favorably with those of a study based on ten years of ISEE data (Verigin et al., 2006). Cluster orbital configuration is ideally designed to study the magnetosheath whose various regions are correctly sampled in our analysis, with a majority of events close to the magnetopause. Note that for the present study the time frame has been extended in comparison with the one in Génot et al. (2009a). A web-based version of the statistical analysis tool developed at CDPP (the French Plasma Physics Data Centre) and used in this study is available at the URL: http://cdpp-amda.cesr.fr. Access is granted upon request (mail to amda@ cesr.fr).

\subsection{Magnetosheath identification}

The first step of our analysis is to determine whether Cluster is located in the magnetosheath. Data are analyzed by $5 \mathrm{~min}$ window: a delay procedure is applied to obtain associated solar wind and IMF parameters from ACE. Shock and magnetopause models, as described by Verigin et al. (2006) and Shue et al. (1997) respectively, are computed dynamically from these parameters in order to locate Cluster as "inside" or "outside" the magnetosheath.

\subsection{Identification and characteristics of mirror-like structures}

The procedure used to determine the presence of mirrorlike structures and the way to characterize them depending on their shape has been extensively exposed in Génot et al. (2009a). For consistency we summarize here the main steps of the procedure but we refer the reader to the previous paper in which instrumental and methodological effects are discussed.

Magnetic field variations associated with mirror modes are almost linearly polarized in the direction of the ambient field. They may be of large amplitude (a few 10\%). From these characteristics, a criterion has been established which follows closely those used by Tátrallyay and Erdös (2002); 
Soucek et al. (2008); Génot et al. (2009a,b); it requires two conditions:

- in order to select a linear polarization with field variations in directions close to that of the ambient magnetic field, the angle between the maximum variance direction and the mean magnetic field is prescribed to be smaller than $20^{\circ}$.

- to prescribe relatively large amplitude, the variance of the field must be larger than $10 \%$.

In order to perform a large statistical survey we use relatively low resolution data $(4 \mathrm{~s})$, which limits the lower sampled mirror event size to $8 \mathrm{~s}$. The above criterion is then applied to all 5 min magnetosheath intervals selected previously. The mean magnetic field is calculated on 10 min window, and the Minimum Variance Analysis (MVA (Sonnerup and Cahill, 1967)) is performed on 5 min windows. At this stage, we do not make any restriction on the values of plasma parameters, as we are interested in mirror-like structures appearing above as well as below the linear mirror instability threshold defined below. In particular no constraint has been imposed on the eigenvalues resulting from the MVA (see discussion in Génot et al., 2001). In order to characterize the plasma with respect to the mirror instability, we define the mirror parameter $C_{\mathrm{M}}$ by

$C_{\mathrm{M}}=\beta_{\perp}\left(T_{\perp} / T_{\|}-1\right)$

where the symbols $\perp$ and $\|$ refer to the direction of the ambient magnetic field. $C_{\mathrm{M}}-1$ is then the distance to the mirror instability threshold. The condition $C_{\mathrm{M}}<1\left(C_{\mathrm{M}}>1\right)$ corresponds to mirror stable (unstable) plasma, while $C_{\mathrm{M}}=1$ refers to marginal stability, for which the linear growth rate is zero. Let us note that the above expression is obtained for biMaxwellian distribution functions and evaluated in the lowfrequency, long-wavelength limit of the Vlasov-Maxwell equations for cold electrons (Hall, 1979; Hellinger, 2007). The interested reader is referred to Génot et al. (2009a) for further caution to take due to the specificity of the ion detector.

An automatized detection of the shape of the structures is a prerequisite for any long term analysis. Tátrallyay and Erdös (2005), analyzing 10 years of ISEE-1, used an algorithm searching for symmetrical shapes of magnetic depressions; Joy et al. (2006) proposed a statistical method based on the determination of the background magnetic field level; Soucek et al. (2008) defined the peakness as the skewness of the time series representing the total wavelet content, between two chosen scales, of the original magnetic field fluctuations. Here we compute the skewness (i.e. the normalized third moment) of the distribution of the magnetic fluctuations $(\delta B=B-<B>$, where the average $<>$ is taken on a 10 min window). Like for the peakness, a positive (negative) value reflects a distribution skewed towards higher (smaller) values, which corresponds to an interval dominated by peaks (holes). A vanishing or small value corresponds to sinusoidal-like (symmetric) fluctuations or alternatively to an interval composed of peaks and holes equally distributed. The latter regime is usually associated with transition periods. The method proved to be efficient by showing that it correctly captures peak- or hole-filled intervals identified by visual inspection. Finally let us recall that larger (smaller) values of the skewness are not related to higher (deeper) structures but to a larger deviation from the sinusoidal shape which also translates into the presence of more isolated structures. The main result of Génot et al. (2009a) was to reveal a positive correlation between the skewness and $C_{\mathrm{M}}$ based on a threefold approach combining observations, numerical simulations and theoretical modeling.

\section{Results and discussion}

\subsection{Statistical analysis}

The procedure described above has been performed and the result are summarized on the left column of Fig. 4. The total number of events is above 25000 . As a function of the normalized flowtime $T$ we plot successively the median values of the mirror instability threshold, the magnetic fluctuations (obtained from the standard deviation) and the skewness. The insert in the top panel shows the distribution of mirror events as a function of the normalized flowtime. The variation of these quantities are now compared with results from numerical simulations.

\subsection{Mirror mode dynamics: comparison with hybrid simulations}

By providing a dating time or timing, the flow time, the magnetosheath model allows to gain a dynamical perspective which can directly be compared with simulation results. Indeed direct simulations of the Vlasov-Maxwell equations enables to study the evolution of mirror modes and the formation of coherent structures. Here we shall refer to hybrid simulations in an expanding box (HEB code, see Hellinger and Trávníček, 2005, and references therein). The expanding box simulation models an evolution of a small fraction of the plasma which expands under the effect of the global magnetosheath flow around the magnetospheric cavity. The model replaces the spatial dependence by a temporal one and neglects global inhomogeneities/heat fluxes (physical lengths increase linearly with time). In this way, it provides a selfconsistent means to study the dynamical properties of waves driven by the magnetosheath plasma. The simulation discussed hereafter is described in Trávníček et al. (2007) and also discussed in Génot et al. (2009a). Initial conditions correspond to an homogeneous plasma only weakly unstable with respect to the mirror and proton cyclotron instabilities: $C_{\mathrm{M}}=1.4$. From the simulation the skewness is evaluated from the magnetic fluctuations in the parallel direction. The 

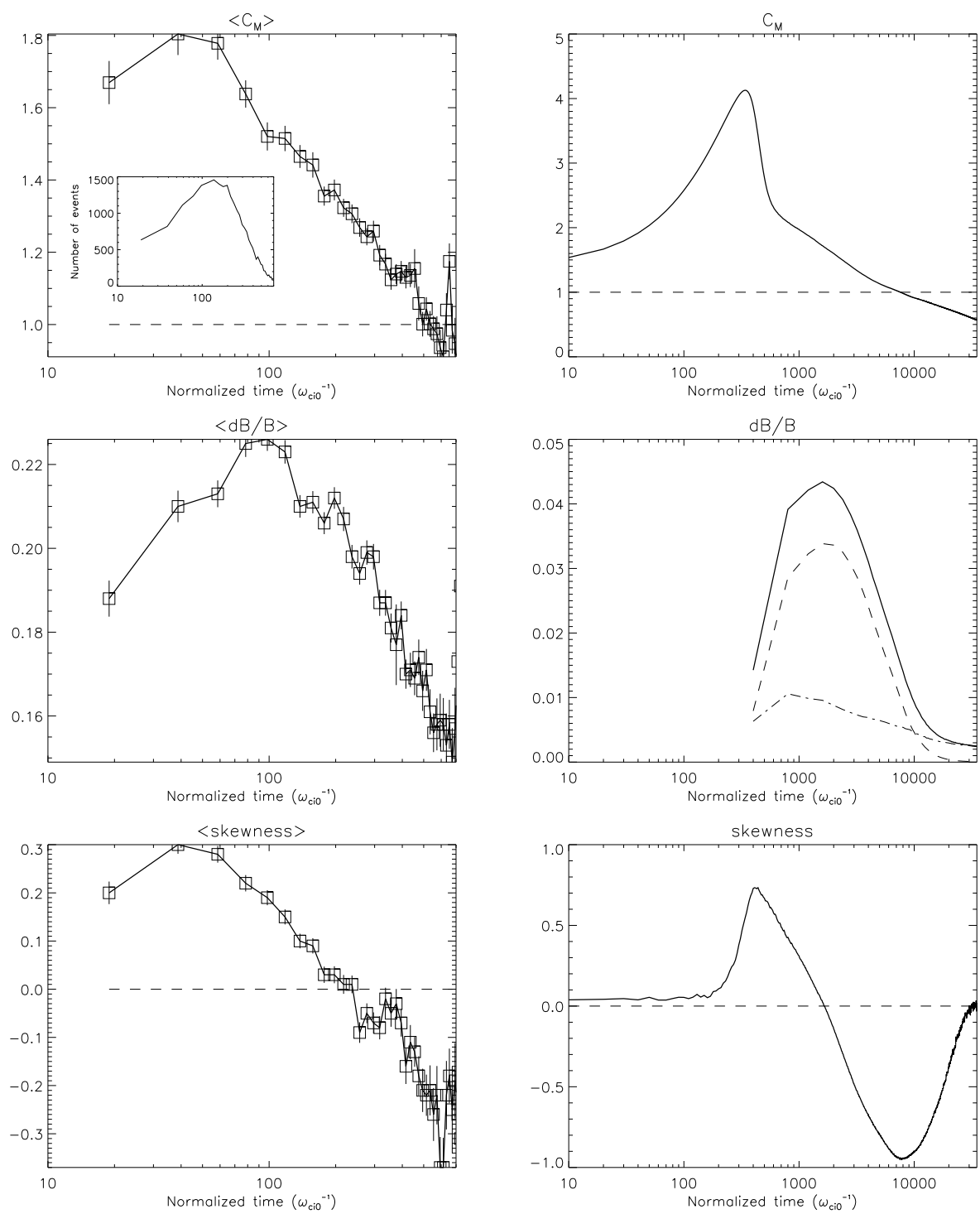

Fig. 4. Left column, observations. As a function of the normalized flow time: (top) median value of $C_{\mathrm{m}}$, (middle) median value of $\delta B / B$, (bottom) median value of the skewness. The error bars are proportional to $\sigma / \sqrt{N}$ where $\sigma$ is the standard deviation and $N$ is the number of mirror events in each flow time bin $(\Delta t=20)$. Right column, simulation. As a function of the normalized time, and averaged in the perpendicular to B direction: (top) $C_{\mathrm{m}}$, (middle) total $\delta B / B$ (solid line), mirror contribution (dash line) and proton cyclotron contribution (dot-dash line), (bottom) skewness of parallel magnetic fluctuations. The insert shows the distribution of mirror events as a function of the normalized flowtime.

modeled quantities equivalent to those obtained from the statistical survey on Cluster data are plotted on the right column of Fig. 4, apart from the wave amplitude fluctuations which are split in contributions from the mirror wave activity and proton cyclotron wave activity together with the total amplitude (second panel, no wave diagnostics were performed before $T \sim 400$ ).

In the following we give a global scenario for the development of mirror structures based on an inter-comparison between simulations and observations illustrated on Fig. 4. At the shock crossing temperature anisotropy is created by the preferential heating of particle in the perpendicular direction such that plasma conditions are well above the mirror threshold ( $C_{\mathrm{M}}$, see top left panel on Fig. 4). Accordingly, mirror structures created at the shock evolve from quasi sinusoidal fluctuations to magnetic peaks. This phase corresponds to the growth of the skewness from small or vanishing to positive values (see bottom panels on Fig. 4). However this early phase is very short living and quickly the peaks are superseded by holes, i.e. the skewness goes back to vanishing and then negative values as plasma conditions get more mirror stable. Both simulations and observations show this distinct 
behaviour. The transition occurs at $T \sim 240$ and $T \sim 1650$ respectively. The physics underlying the transitional phase from peak to hole is not fully understood although some theoretical works have tackled this question: Passot et al. (2006) invoked energetics considerations in an anisotropic MHD model, whereas a dynamical kinetic model has been proposed by Kuznetsov et al. (2007) (see also the discussion in Génot et al., 2009a).

During the early phase total and mirror amplitudes from the simulation quickly increase and saturate. Similarly to observations this saturation occurs when the skewness goes to vanishing values, i.e. when unstable peaks are less and less present to the expense of magnetic holes. After vanishing the skewness remains negative corresponding to the long-term predominance of magnetic holes both in the simulation box and in the magnetosheath. In the simulation a trend, not clearly observed in the statistical survey, shows a reversal toward less negative skewness. This occurs when the plasma turns stable with respect to the mirror instability $\left(C_{\mathrm{M}}<1\right)$; accordingly the mirror wave activity decreases and is superseded by proton cyclotron wave activity as it is illustrated on the second panel of the right column of Fig. 4. This behaviour (the progressive prominence of IC waves) may be extrapolated to the magnetosheath itself but the statistics at large $T$ is not sufficient to conclude. In conclusion, for the later phase of the simulation or for long flow times which correspond to magnetosheath observations on the flanks or close to the magnetopause, the plasma essentially turns stable $\left(C_{\mathrm{M}}<1\right)$ which is followed by a decrease of the fluctuation amplitude, i.e. mirror holes are slowly damped. The existence of mirror structures for mirror stable plasma conditions has been explained by a bistability phenomenon (Baumgärtel, 2001; Passot et al., 2006). It was shown that in such conditions only magnetic holes survive; this explains why magnetic holes may be observed isolated whereas magnetic peaks are observed in trains (filling the whole unstable plasma volume).

Despite the good similarities in the general behaviour of the simulation and observational approaches, a difference remains concerning the temporal development of the mirror instability process. The simulation time scale is indeed about an order of magnitude larger than the observational one. The main reason is that the simulation setting was performed (in the original work Trávníček et al., 2007) with the main goal of having a good separation of expansion and proton kinetic time scales. In any case, a precise quantification of the real expansion time is a difficult task. According to these constraints the expansion time of the mimicked magnetosheath $\left(t_{\mathrm{e}}\right.$, see also the Appendix B) was chosen to be on the order of the inverse of the maximum growth rate of the proton instabilities (about 1000 inverse proton gyrofrequencies, $t_{\mathrm{e}} \simeq$ $\gamma^{-1} \simeq 1000 \Omega_{\mathrm{p}}^{-1}$ ). The comparison with observations shows that this choice, albeit convenient for simulation purposes, is probably not the more appropriate to mimic the real magnetosheath. However other factors come into play which pre- vent from expecting a close simulation/observations match only by adjusting the expansion time parameter: observations are analyzed in a statistical manner where as the simulation is presented for a given set-up; furthermore, in the simulation there is no real shock, solar wind turbulence, alpha particles, .... In conclusion the comparison can only be qualitative based on the global variations.

The relatively quick saturation of mirror fluctuations evidenced here in data and simulation differs from the conclusion by Tsurutani et al. (2011). Indeed these authors claimed that no saturation is evidenced inside the magnetosheath and that the mirror fluctuations reach their maximum amplitudes near the magnetopause. This should be nuanced. In the statistical work by Génot et al. (2009b) it was shown that the largest mirror fluctuations were indeed observed in the middle magnetosheath (as peaks), at about a fractional distance of one third from the magnetopause. This observation is consistent with the saturation process reported here; and also in Bavassano Cattaneo et al. (1998) which reports continuous observations of mirror structures throughout the Kronian magnetosheath, evolving from quasi-sinusoidal oscillations at the shock, towards peaks and finally holes near the magnetopause. A major difference between our analysis and those of Tsurutani et al. (2011) and Tátrallyay and Erdös (2005) (among other) is that we are considering both peak and hole structures whereas the above cited works only deals with magnetic dips or magnetic decreases (MD). This difference and its consequences have already been discussed in Génot et al. (2009b), in particular through their Fig. 5 which shows the distribution of $C_{\mathrm{M}}$ in a plane fractional distance - zenith angle. In agreement with the present study it illustrates the fact that the most mirror-unstable region is the middle magnetosheath (mostly filled by magnetic peaks). The large free energy available there is a probable consequence of an additive combination of effects: shock compression and field line draping. Even though peaks are short living structures (as simulations and observations show), they occur under the most mirror unstable plasma conditions; therefore any statistical survey on mirror events should encompass all structural shapes in order to approach a full understanding. Finally the ambivalent role of field line draping must be evoked. Indeed the field line draping at the subsolar magnetopause is certainly a cause of increased anisotropy (this is one conclusion of Tátrallyay and Erdös, 2005, based on a work by Zwan and Wolf, 1996) but it also a cause of decreasing $\beta$ through the increase of the magnetic field and the decrease of the density in the PDL. Therefore the plasma conditions are often stable with respect to the mirror instability and the large amplitude magnetic holes observed there are most probably explained by the bi-stability process described in Passot et al. (2006) and Kuznetsov et al. (2007).

The preceding description can also be envisaged from analytical considerations also used in the HEB model. Immediately after the shock crossing, the anisotropy increases whatever the location is in the magnetosheath (subsolar region 
or flank). This is what is observed in the simulation and in observations although the observed anisotropy only slightly increases after the wave saturation is reached, $T \sim 100$ (for reference $T=100$ corresponds to about $65 \mathrm{~s}$; see below). Indeed, in the case of a slow perturbation one expects that the first and the second adiabatic invariants will be conserved as long as wave activity is negligible. This perturbation may be compression (subsolar region case) or expansion (flank magnetosheath case): in both cases it can be showed that anisotropy increases (see Appendix B) whereas the plasma $\beta$ decreases. This translates into an increase of the mirror threshold which is readily observed in the early phase of the mirror growth, at least until a normalized flowtime $T \sim 40$ in observations and $T \sim 350$ in the simulation which corresponds to the stage of wave activity growth. Then the mirror threshold $C_{\mathrm{M}}$ decreases to reach stable plasma conditions at longer times (the behaviour of the mirror instability threshold $C_{\mathrm{M}}$ is in agreement with the one described for the skewness as both quantities are statistically positively correlated). It is possible to postulate that this behavior is due to the growth of waves which become of sufficiently high amplitudes such that the wave-free description presented in Appendix B is no longer valid: invariants are not longer conserved and the simple description of the variations of the anisotropy and $\beta$ obtained from analytical conservation does not hold. This also expresses the fact that the free energy contained in the anisotropy is consumed by the wave activity produced by instabilities.

In conclusion simulations and observational results (the latter being associated to the magnetosheath flow model) show strong similarities in the general behaviour of characteristic parameters tracking the mirror instability evolution.

\subsection{Comparison with Soucek et al. (2008) in the magnetosheath}

The evolution of the mirror threshold in our study may be compared to the behaviour described in Fig. 9 of Soucek et al. (2008): as a function of $X_{\mathrm{GSE}}\left(-4 R_{\mathrm{E}} \leq X_{\mathrm{GSE}} \leq 8 R_{\mathrm{E}}\right.$, corresponding to 2 months of Cluster 1 data) their statistical parameter $R$ (equivalent to our parameter $C_{\mathrm{M}}$ ) slowly decreases to reach below one values in the night side (for $X_{\mathrm{GSE}} \simeq-3 R_{\mathrm{E}}$ ). In our analysis, the normalized flow time for which this transition occurs is $T \sim 550$ : this translates to $380 \mathrm{~s}$ if one assumes an average magnetosheath magnetic field amplitude of $15 \mathrm{nT}$. A $200 \mathrm{~km} \mathrm{~s}^{-1}$ plasma flow would then correspond to a propagation of $12 R_{\mathrm{E}}$ inside the magnetosheath from the shock. This indeed also corresponds to the night side of the magnetosphere for stream lines not originating too close to the subsolar region.

\subsection{Comparison with observations at Jupiter}

Similar to our dynamical approach is the work by Joy et al. (2006) in which observed mirror structures (also character- ized in hole/peak categories, with a different scheme though) are positioned in a simulated Jovian magnetosheath (from a global MHD simulation by Ogino et al., 1998). Flow times are computed along stream lines and show that peak structures (observed by Voyager 1, Galileo and Pioneer 11) are mainly seen close to the shock whereas observations further down the magnetosheath (observed by Galileo, Pioneer 11 and Ulysses) show magnetic holes. Geometrical considerations in normalized (to the planetary radius) units lead to think that the peak/hole transition occurs at the same average location in both Earth's and Jupiter's magnetosheaths. The question remains whether the flow time may also be relatively compared. One should therefore consider how to scale Jovian observations with respect to Earth's. We identify the following ratios (Jupiter/Earth):

$$
\begin{aligned}
\text { - } & \sim 11 \text { in the planetary radius: } 71490 / 6380(\mathrm{~km}), \\
\text { - } & \sim 10 \text { in the magnetosheath size: } 40 / 4 \text { (planetary radii), } \\
\text { - } & \sim 1 / 5 \text { in the magnetosheath magnetic field amplitude: } \\
& 3 / 15(\mathrm{nT})
\end{aligned}
$$

The Jupiter data scaled in the MHD simulation show that the peak/hole transition occurs in the range 30-40 h. Comparing this to the $170 \mathrm{~s}$ in the Earth's case (obtained from a transition at $T \sim 240$ and mean magnetic field value of $15 \mathrm{nT}$ ) yields a ratio of $\sim 1000$ for the time scale. The mirror instability dynamics (via its growth phase) is scaled by the cyclotron frequency, at least in the linear phase. From the ratios above, the growth is expected to be five times slower in Jupiter's magnetosheath. The peak/hole transition on the contrary is governed by changes in local plasma conditions ( $\beta$ and anisotropy, or $C_{\mathrm{M}}$ ) which are themselves governed by the MHD flow around the magnetospheric obstacle (considering the solar wind to be identical). Timescales are therefore planetary and not plasma. However one should take into account the fact that the Jovian magnetosheath flow is slower than on Earth. This comes from the higher compressibility of the magnetosphere due to the non negligible role of the magnetospheric plasma in controlling the internal pressure. This effect induces the possibility for reduced (and even sunward) flows into the magnetosheath which have a global decelerating effect. From the simulation by Ogino et al. (1998) and computed flowtimes one deduces an average speed of $50 \mathrm{~km} \mathrm{~s}^{-1}$ which is a factor $\sim 4$ less than for the Earth case. From scaling considerations together with this mean flow evaluation we arrive to a factor 440 (to be compared to the factor 1000 cited above), i.e. to a typical peak/hole transition flowtime of $20 \mathrm{~h}$ (instead of 30-40 h). From the uncertainties and different approximations made in this coarse comparison we conclude that, despite large differences in the characteristic length scales and plasma environments of Earth's and Jupiter's magnetosheaths, the mirror peak/hole transition occurs at similar (normalized) locations. These locations are constrained by the large scale evolution of plasma parameters in the magnetosheath flow. 
It should also be noted that the mirror structures observed in both environments have a similar characteristic length scales of 20-25 proton gyro-radii (Tsurutani et al., 1982).

\section{Conclusions}

In this paper it was shown how to build and use a simple analytic model of magnetosheath magnetic and velocity fields in order to obtain the timing of mirror structures, i.e. their "age" since their formation. It is assumed that these structures are formed at the bow shock by the temperature anisotropy which naturally develops there. The analysis of a database of mirror events covering almost 10 years of Cluster data shows that magnetic peaks are young structures whereas magnetic holes are relatively aged structures, a result which was already obtained from numerical simulations. The model helped to gain the dynamical perspective absent from sole in-flight measurements.

The present study also emphasizes the fact that both magnetic peaks and holes must be considered as equally important elements of the same evolutionary process. In order to understand mirror mode physics, studying only one type of structures and neglecting others may lead to partial or erroneous conclusions.

A coarse comparison with data from several crossings of the Jovian magnetosheath revealed that the locations of the peak-to-hole transition is located in similar regions than in the Earth case when normalized quantities (magnetosheath sizes, flows, ...) are considered. If the ubiquitous nature of the mirror mode micro-physics was already assessed, the large scale communality in the evolution of these waves is possibly illustrated here. The dynamical scenario described in this paper may also apply to other environments where mirror waves were observed. For instance, and consistently with our scenario, Burlaga et al. (2006) reported the presence of magnetic humps behind the termination shock crossed by Voyager 1 whereas magnetic holes were observed later and further down in the heliosheath.

\section{Appendix A}

\section{Rankine-Hugoniot relations}

Once the location of the shock has been determined by tracking back the flow lines (the velocity magnitude is not required for this operation), the normal to the shock ( $\boldsymbol{n}=$ $\left.\left(n_{\mathrm{x}}, n_{\mathrm{y}}, n_{\mathrm{z}}\right)\right)$ is computed from the KF model. In order to apply the transformation between the upstream/downstream reference frame $(\boldsymbol{x}, \boldsymbol{y}, \boldsymbol{z})$ and the normal/tangential $(\boldsymbol{n}, \boldsymbol{t})$ one in which the Rankine-Hugoniot relations are expressed, polar and azimuthal angles are defined ( $\theta$ and $\phi$ respectively). Transforming $\boldsymbol{x}$ into $\boldsymbol{n}$ gives the following relations:

$\tan \theta=\frac{n_{\mathrm{z}}}{\left(n_{\mathrm{x}}^{2}+n_{\mathrm{y}}^{2}\right)^{1 / 2}}$ and

$\tan \phi=\frac{n_{\mathrm{y}}}{n_{\mathrm{x}}}$

The incident velocity $\boldsymbol{v}_{1}$ is first transformed into the normal/tangential reference frame. We then apply the RankineHugoniot relations $\rho_{1} v_{n 1}=\rho_{2} v_{n 2}$ and $v_{t 1} \simeq v_{t 2}$ (this is commonly used but justified below) and rotate the velocity back to the original reference frame. The $\mathrm{x}$-component of the downstream velocity is finally given by

$$
\begin{aligned}
v_{2 \mathrm{x}}= & \rho_{1} / \rho_{2} \cos \theta \cos \phi\left[\cos \phi\left(v_{1 \mathrm{x}} \cos \theta-v_{1 \mathrm{z}} \sin \theta\right)-v_{1 \mathrm{y}} \sin \phi\right] \\
& +\cos \theta \sin \phi\left[\sin \phi\left(v_{1 \mathrm{x}} \cos \theta-v_{1 \mathrm{z}} \sin \theta\right)+v_{1 \mathrm{y}} \cos \phi\right](\mathrm{A} 3)
\end{aligned}
$$

where $\left(v_{1 \mathrm{x}}, v_{1 \mathrm{y}}, v_{1 \mathrm{z}}\right)$ is the incident solar wind velocity vector. As $v_{1 \mathrm{x}} \gg v_{1 \mathrm{y}}, v_{1 \mathrm{z}}$ this relation essentially reduces to

$v_{2 \mathrm{x}} \simeq v_{1 \mathrm{x}} \cos ^{2} \theta\left(1+\left(\rho_{1} / \rho_{2}-1\right) \cos ^{2} \phi\right)$

Similarly $v_{2 \mathrm{y}}$ and $v_{2 \mathrm{z}}$ may be obtained.

$v_{2 \mathrm{x}}$ is the downstream velocity component which enters into the evaluation of the parameter $C$ given in Eq. (4). Another parameter which needs to be determined is the density contrast $R=\rho_{2} / \rho_{1}$. Its exact value has however a minor influence on our modeled velocity magnitude. The exact approach is derived from the main assumption, i.e. that the velocity flow lines are the magnetic field lines of the KF model for $\boldsymbol{B}_{\mathrm{IMF}}=\left(B_{\mathrm{X}}, 0,0\right)_{\mathrm{GSE}}$ which may be written

$\frac{v_{\mathrm{z}}}{v_{\mathrm{x}}}=\frac{B_{\mathrm{z}}}{B_{\mathrm{X}}}$

where quantities are taken in the magnetosheath. When this is evaluated just downstream of the shock, Eqs. (A4) and (A5) may be used together with the KF model value of $\frac{B_{\mathrm{Z}}}{B_{\mathrm{x}}}$ to solve for $R$; one shows that

$\frac{1}{R}=1-\frac{\frac{B_{\mathrm{Z}}}{B_{\mathrm{X}} \cos ^{2} \phi}}{\frac{B_{\mathrm{Z}} \cos ^{2} \theta}{B_{\mathrm{X}}}-\cos \theta \sin \theta}$

Equation (A6) enables to account for the changes in the density ratio with the position on the shock surface. Without large discrepancies in the velocity magnitude it is alternatively possible to use an empirically value derived from gas dynamics models and which depends on the actual stand off distances of the magnetopause and bow shock (see for instance Spreiter et al., 1966)

$R=\frac{1.1 R_{\mathrm{mp}}}{R_{\mathrm{bs}}-R_{\mathrm{mp}}}$

Finally, let us evaluate the validity of the approximation $v_{t 1} \simeq v_{t 2}$ used above. Rearranging exact Rankine-Hugoniot relations (see for instance Génot, 2008; Génot, 2009) it is possible to show that

$\boldsymbol{v}_{t 2}=\boldsymbol{v}_{t 1}+v_{n 1} \frac{1-1 / R}{\frac{M_{\mathrm{a}}^{2}}{R \cos ^{2} \theta_{\mathrm{Bn}}}-1} \tan \theta_{\mathrm{Bn}} \boldsymbol{t}$ 
or

$v_{t 2} / v_{n 1}=\tan \theta_{\mathrm{Vn}}+\frac{1-1 / R}{\frac{M_{\mathrm{a}}^{2}}{R \cos ^{2} \theta_{\mathrm{Bn}}}-1} \tan \theta_{\mathrm{Bn}}$

where $\theta_{\mathrm{Vn}}$ is the angle between the upstream velocity vector and the shock normal. For typical values $(R=4$, upstream $\left.M_{\mathrm{a}}=10\right)$ the factor $\frac{1-1 / R}{\frac{M_{\mathrm{a}}^{2}}{R \cos ^{2} \theta_{\mathrm{Bn}}}-1} \tan \theta_{\mathrm{Bn}}$ maximizes at 0.015 as a function of $\theta_{\mathrm{Bn}}$. Therefore for $\theta_{\mathrm{Vn}} \gtrsim 2^{\circ}$ the second term above is negligible in front of $\tan \theta_{\mathrm{Vn}}$ and the approximation $\boldsymbol{v}_{t 2}=\boldsymbol{v}_{t 1}$ is valid (this angle increases to $12^{\circ}$ for low Mach number, i.e. $M_{\mathrm{a}}=3$, and decreases with $R$ ). Practically this approximation fails only in a restricted region close to the subsolar point.

\section{Appendix B}

\section{Compression/expansion in the magnetosheath}

Depending on the region in the magnetosheath, the plasma will encounter either a compression (subsolar region) or an expansion (on the flanks). In the following we estimate the variations of relevant parameters. This approach was used in order to introduce concepts of the HEB code in which are implemented compressed or expanding simulation domains.

During transformation the coordinates $\boldsymbol{x}$ are modified such that

$x \rightarrow \bar{L} x$

\section{B1 Expansion}

It acts (equivalently) in two directions: one parallel and one perpendicular to the magnetic field (see Trávníček et al., $2007)$, i.e. $\operatorname{diag}(\bar{L})=\left(L_{\mathrm{e}}, L_{\mathrm{e}}, 1\right)$ with

$L_{\mathrm{e}}=1+\frac{t}{t_{\mathrm{e}}}$

From the conservation of the first and second invariants, one gets

$\frac{T_{\perp}}{T_{\|}} \propto L_{\mathrm{e}}$

$\beta_{\|} \propto \frac{1}{L_{\mathrm{e}}^{2}}$

\section{B2 Compression}

It acts in one direction perpendicular to the magnetic field (along the magnetopause normal direction, see Hellinger and Trávníček, 2005), i.e. $\operatorname{diag}(\bar{L})=\left(1, L_{\mathrm{c}}, 1\right)$ with

$L_{\mathrm{c}}=1-\frac{t}{t_{\mathrm{c}}}$
From the conservation of the first and second invariants, one gets

$\frac{T_{\perp}}{T_{\|}} \propto \frac{1}{L_{\mathrm{c}}}$

$\beta_{\|} \propto L_{\mathrm{c}}$

\section{B3 Conclusion}

From the above results, for both transformations, the anisotropy and the mirror threshold increase with time, and $\beta_{\|}$decreases, as long as the wave activity is negligible.

Acknowledgements. V.G. thanks the CDPP (http://cdpp.cesr.fr) team members for their efforts in developing the software used in this study in particular the online analysis tool AMDA (http: //cdpp-amda.cesr.fr). V.G. also thanks N. André (IRAP) for useful discussions.

Guest Editor M. Taylor thanks T. V. Laitinen and another anonymous referee for their help in evaluating this paper.

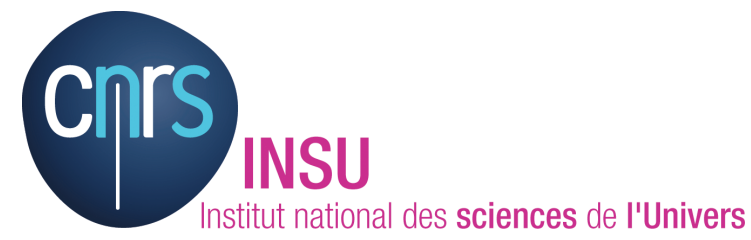

The publication of this article is financed by CNRS-INSU.

\section{References}

Balogh, A., Carr, C. M., Acuña, M. H., Dunlop, M. W., Beek, T. J., Brown, P., Fornacon, K.-H., Georgescu, E., Glassmeier, K.H., Harris, J., Musmann, G., Oddy, T., and Schwingenschuh, K.: The Cluster Magnetic Field Investigation: overview of in-flight performance and initial results, Ann. Geophys., 19, 1207-1217, doi:10.5194/angeo-19-1207-2001, 2001.

Baumgärtel, K.: Fluid approach to mirror mode structures, Planet. Space. Sci., 49, 1239-1247, 2001.

Bavassano Cattaneo, M. B., Basile, C., Moreno, G., and Richardson, J. D.: Evolution of mirror structures in the magnetosheath of Saturn from the bow shock to the magnetopause, J. Geophys. Res., 103, 11961-11972, 1998.

Burlaga, L. F., Ness, N. F., and Acũna, M. H.: Trains of magnetic holes and magnetic humps in the heliosheath, Geophys. Res. Lett., 33, L21106, doi:10.1029/2006GL027276, 2006.

Califano, F., Hellinger, P., Kuznetsov, E., Passot, T., Sulem, P. L., and Travnicek, P.: Nonlinear mirror mode dynamics: simulations and modeling, J. Geophys. Res., 113, A08219, doi:10.1029/2007JA012898, 2008.

Fazakerley, A. N. and Southwood, D. J.: Mirror instability in the magnetosheath, Adv. Space Res., 14, 65-68, 1994.

Gary, S. P., Fuselier, S. A., and Anderson, B. J.: Ion anisotropy instabilities in the magnetosheath, J. Geophys. Res., 98, 14811488, doi:10.1029/92JA01844, 1993.

Génot, V.: Mirror and firehose instabilities in the heliosheath, The Astrophysical Journal, 687, L119-L122, 2008. 
Génot, V.: Analytical solutions for anisotropic MHD shocks, Astrophysics and Space Sciences Transactions, 5, 31-34, 2009.

Génot, V., Schwartz, S. J., Mazelle, C., Balikhin, M., Dunlop, M., and Bauer, T. M.: Kinetic study of the mirror mode, J. Geophys. Res., 106, 21611-21622, doi:10.1029/2000JA000457, 2001.

Génot, V., Budnik, E., Hellinger, P., Passot, T., Belmont, G., Trávníček, P. M., Sulem, P.-L., Lucek, E., and Dandouras, I.: Mirror structures above and below the linear instability threshold: Cluster observations, fluid model and hybrid simulations, Ann. Geophys., 27, 601-615, doi:10.5194/angeo-27-601-2009, 2009a.

Génot, V., Budnik, E., Jacquey, C., Dandouras, I., and Lucek, E.: Mirror modes observed with Cluster in the Earth's magnetosheath: statistical study and IMF/solar wind dependence, Adv. Geosci., volume 14: Solar Terrestrial (ST), edited by: Duldig, M., Singapore: World Scientific, p. 263, 2009b.

Hall, A. N.: Finite ion Larmor radius modifications to the firehose and mirror instabilities, J. Plasma Phys., 21, 431-443, 1979.

Hasegawa, A.: Drift mirror instability of the magnetosphere, Phys. Fluids, 12, 2642-2650, 1969.

Hau, L.-N., Wang, B.-J., and Teh, W.-L.: Slow mode waves and mirror instability in gyrotropic Hall magnetohydrodynamic model, Phys. Plasmas, 12, 122904-122904-8, 2005.

Hellinger, P.: Comment on the linear mirror instability near the threshold, Phys. Plasmas, 14, 8, 082105-082105-4, 2007.

Hellinger P. and Trávníček, P.: Magnetosheath compression: Role of characteristic compression time, alpha particle abundance, and alpha/proton relative velocity, J. Geophys. Res., 110, A04210, doi:10.1029/2004JA010687, 2005.

Horbury, T. S., Lucek, E. A., Balogh, A., Dandouras, I., and Rème, H.: Motion and orientation of magnetic field dips and peaks in the terrestrial magnetosheath, J. Geophys. Res., 109, 9209, doi:10.1029/2003JA010237, 2004.

Joy, S. P., Kivelson, M. G., Walker, R. J., Khurana, K. K., Russell, C. T., and Paterson, W. R.: Mirror mode structures in the Jovian magnetosheath, J. Geophys. Res., 111, 12212, doi:10.1029/2006JA011985, 2006.

Kallio, E. J. and Koskinen, H. E. J.: A semiempirical magnetosheath model to analyze the solar wind-magnetosphere interaction, J. Geophys. Res., 105, 27469-27480, 2000.

Kaufmann, R. L., Horng, J.-T., and Wolfe, A.: Large-amplitude hydromagnetic waves in the inner magnetosheath, J. Geophys. Res., 75, 4666-4676, 1970.

Kobel, E. and Flückiger, E. O.: A model of the steady state magnetic field in the magnetosheath, J. Geophys. Res., 99, 23617-23622, 1994.

Kuznetsov, E. A., Passot, T., and Sulem, P. L.: Dynamical model for nonlinear mirror modes near threshold, Phys. Rev. Lett., 98, 235003, 2007.

Lucek, E. A., Dunlop, M. W., Horbury, T. S., Balogh, A., Brown, P., Cargill, P., Carr, C., Fornaçon, K.-H., Georgescu, E., and Oddy, T.: Cluster magnetic field observations in the magnetosheath: four-point measurements of mirror structures, Ann. Geophys., 19, 1421-1428, doi:10.5194/angeo-19-1421-2001, 2001.

Ogino, T., Walker, R. J., and Kivelson, M. G.: A global magnetohydrodynamic simulation of the Jovian magnetosphere, J. Geophys. Res., 103, 225-235, 1998.

Paschmann, G., Sckopke, N., Haerendel, G., Papamastorakis, J., Bame, S. J., Asbridge, J. R., Gosling, J. T., Hones Jr., E. W., and Tech, E. R.: ISEE plasma observations near the subsolar magnetopause, Space Sci. Rev., 22, 717-737, 1978.

Passot T., Ruban, V., and Sulem, P. L.: Fluid description of trains of stationary mirror structures in a magnetized plasma, Phys. Plasmas, 13, 102310-102310-10, doi:10.1063/1.2356485, 2006.

Rème, H., Aoustin, C., Bosqued, J. M., Dandouras, I., Lavraud, B., Sauvaud, J. A., Barthe, A., Bouyssou, J., Camus, Th., CoeurJoly, O., Cros, A., Cuvilo, J., Ducay, F., Garbarowitz, Y., Medale, J. L., Penou, E., Perrier, H., Romefort, D., Rouzaud, J., Vallat, C., Alcaydé, D., Jacquey, C., Mazelle, C., d’Uston, C., Möbius, E., Kistler, L. M., Crocker, K., Granoff, M., Mouikis, C., Popecki, M., Vosbury, M., Klecker, B., Hovestadt, D., Kucharek, H., Kuenneth, E., Paschmann, G., Scholer, M., Sckopke, N., Seidenschwang, E., Carlson, C. W., Curtis, D. W., Ingraham, C., Lin, R. P., McFadden, J. P., Parks, G. K., Phan, T., Formisano, V., Amata, E., Bavassano-Cattaneo, M. B., Baldetti, P., Bruno, R., Chionchio, G., Di Lellis, A., Marcucci, M. F., Pallocchia, G., Korth, A., Daly, P. W., Graeve, B., Rosenbauer, H., Vasyliunas, V., McCarthy, M., Wilber, M., Eliasson, L., Lundin, R., Olsen, S., Shelley, E. G., Fuselier, S., Ghielmetti, A. G., Lennartsson, W., Escoubet, C. P., Balsiger, H., Friedel, R., Cao, J.-B., Kovrazhkin, R. A., Papamastorakis, I., Pellat, R., Scudder, J., and Sonnerup, B.: First multispacecraft ion measurements in and near the Earth's magnetosphere with the identical Cluster ion spectrometry (CIS) experiment, Ann. Geophys., 19, 1303-1354, doi:10.5194/angeo19-1303-2001, 2001.

Romashets, E. P., Poedts, S., and Vandas, M.: Modeling of the magnetic field in the magnetosheath region, J. Geophys. Res., 113 A02203, doi:10.1029/2006JA012072, 2008.

Schwartz, S. J., Burgess, D., and Moses, J. J.: Low-frequency waves in the Earth's magnetosheath: present status, Ann. Geophys., 14, 1134-1150, doi:10.1007/s00585-996-1134-z, 1996.

Shue, J.-H., Chao, J. K., Fu, H. C., Russell, C. T., Song, P., Khurana, K. K., and Singer, H. J.: A new functional form to study the solar wind control of the magnetopause size and shape, J. Geophys. Res., 102, 9497-9512, 1997.

Sonnerup, B. U. O. and Cahill Jr., L. J.: Magnetopause structure and attitude from Explorer 12 observations, J. Geophys. Res., 72, 171-183, 1967.

Soucek, J., Lucek, E., and Dandouras, I.: Properties of magnetosheath mirror modes observed by Cluster and their response to changes in plasma parameters, J. Geophys. Res., 113, A04203, doi:10.1029/2007JA012649, 2008.

Spreiter, J. R., Summers, A. L., and Alksne, A. Y.: Hydromagnetic flow around the magnetosphere, Planet. Space Sci., 14, 223-250, 1966.

Stasiewicz, K.: Theory and observations of slow-mode solitons in space plasmas, Phys. Rev. Lett., 93, 125004, doi:10.1103/PhysRevLett.93.125004, 2004.

Tátrallyay, M. and Erdös, G.: The evolution of mirror mode fluctuations in the terrestrial magnetosheath, Planet. Space Sci., 50, 593-599, 2002.

Tátrallyay, M. and Erdös, G.: Statistical investigation of mirror type magnetic field depressions observed by ISEE-1, Planet. Space Sci., 53, 33-40, 2005.

Tátrallyay, M., Erdös, G., Balogh, A., and Dandouras, I.: The evolution of mirror type magnetic fluctuations in the magnetosheath based on multipoint observations, Adv. Space Res., 41, 15371544, 2008. 
Trávníček, P., Hellinger, P., Taylor, M. G. G. T., Escoubet, C. P., Dandouras, I., and Lucek, E.: Magnetosheath plasma expansion: hybrid simulations, Geophys. Res. Lett., 34, L15104, doi:10.1029/2007GL029728, 2007.

Tsurutani, B. T., Smith, E. J., Anderson, R. R., Ogilvie, K. W., Scudder, J. D., Baker, D. N., and Bame, S. J.: Lion roars and nonoscillatory drift mirror waves in the magnetosheath, J. Geophys. Res., 87, 6060-6072, 1982.

Tsurutani, B. T., Lakhina, G. S., Verkhoglyadova, O. P., Echer, E., Guarnieri, F. L., Narita, Y., and Constantinescu, D. O.: Magnetosheath and heliosheath mirror mode structures, interplanetary magnetic decreases, and linear magnetic decreases: Differences and distinguishing features, J. Geophys. Res., 116, A02103, doi:10.1029/2010JA015913, 2011.
Verigin, M. I., Tátrallyay, M., Erdös, G., and Kotova, G. A.: Magnetosheath-Interplanetary medium reference frame: Application for a statistical study of mirror type waves in the terrestrial plasma environment, Adv. Space Res., 37, 515-521, 2006.

Zwan, B. J. and Wolf, R. A.: Depletion of the solar wind plasma near a planetary boundary, J. Geophys. Res., 81, 1636-1648, doi:10.1029/JA081i010p01636, 1976. 